\title{
Investigation of future climate change over the British Isles using weather patterns
}

\author{
James O. Pope ${ }^{1}$ (D) $\cdot$ Kate Brown $^{1} \cdot$ Fai Fung $^{1} \cdot$ Helen M. Hanlon $^{1} \cdot$ Robert Neal $^{1} \cdot$ Erika J. Palin $^{1} \cdot$ Anne Reid $^{1}$
}

Received: 3 February 2021 / Accepted: 13 October 2021 / Published online: 5 November 2021

(c) The Author(s) 2021

\begin{abstract}
For those involved in planning for regional and local scale changes in future climate, there is a requirement for climate information to be available in a context more usually associated with meteorological timescales. Here we combine a tool used in numerical weather prediction, the 30 weather patterns produced by the Met Office, which are already applied operationally to numerical weather prediction models, to assess changes in the UK Climate Projections (UKCP) Global ensemble. Through assessing projected changes in the frequency of the weather patterns at the end of the 21 st Century, we determine that future changes in large-scale circulation tend towards an increase in winter of weather patterns associated with cyclonic and westerly wind conditions at the expense of more anticyclonic, settled/blocked weather patterns. In summer, the results indicate a shift towards an increase in dry settled weather types with a corresponding reduction in the wet and windy weather types. Climatologically this suggests a shift towards warmer, wetter winters and warmer, drier summers; which is consistent with the headline findings from the UK Climate Projections 2018. This paper represents the first evaluation of weather patterns analysis within UKCP Global. It provides a detailed assessment of the changes in these weather patterns through the 21st Century and how uncertainty in emissions, structural and perturbed parameters affects these results. We show that the use of these weather patterns in tandem with the UKCP projections is useful for future work investigating changes in a range of weather-related climate features such as extreme precipitation.
\end{abstract}

\section{Introduction}

The increasing temporal and spatial resolution of climate models is enabling models to resolve smaller, more intense mesoscale weather features; opening up the opportunity for assessments of how changes in climate will influence the weather. Sectors and risks such as: agriculture, coastal flooding, drought, power distribution, public health, transport and urban planning (e.g. Huang et al. 2020; Macintyre et al. 2018; Macintyre and Heaviside 2019; Neal et al. 2018; Osbourne and Evans 2019; Palin et al. 2013; 2016; Panteli and Mancarella 2015; Pregnolato et al. 2016; Richardson et al. 2017) are all greatly influenced by the frequency of extremes in temperature, precipitation and/ or wind speed on fine temporal and/or spatial scales. However, there is still a disconnect between the resolution of climate or Earth system models and the resolution required

James O. Pope

james.pope@metoffice.gov.uk

1 Met Office, Fitzroy Road, Exeter EX1 3PB, Devon, UK for these sectors, limiting the applicability of climate model projections. An alternative approach could be to utilise the distribution of large-scale circulation features, given these meteorological variables are governed by the large-scale circulation patterns which drive the type of weather a particular region experiences; we can explore whether projected changes in weather patterns could potentially be used to understand future climate hazards. Within this analysis, a weather pattern refers to a specific daily circulation pattern over a defined geographical domain (Neal et al. 2016). Quantifying patterns of largescale circulation into weather patterns or regimes have been used for a number of decades in weather forecasting for example the Lamb weather types (Lamb 1972) or to assess the climatology of mesoscale events such as marine cold air outbreaks (i.e. Papritz and Grams 2018). The Met Office developed a set of 30 weather patterns representative of circulation over the North Atlantic-European domain. These patterns are used operationally as part of a probabilistic medium- to long-range weather pattern forecasting tool (Neal et al. 2016). By objectively assigning ensemble members from a global ensemble prediction 
system (EPS) to their nearest circulation type, a probabilistic forecast can be produced and used as an operational forecasting guidance tool. These forecasts can also be verified allowing us to understand the predictability of different circulation types as forecast through numerical weather prediction (NWP) models (Ferranti et al. 2015; Neal et al. 2016). The weather patterns have also been used as an application to understand risk, such as the risk of a coastal flooding event (Neal et al. 2018). By combining information on tides with weather patterns and a coastal flooding model, a forecast tool for medium- to long-range flood event likelihood was produced (Neal et al. 2018).

In this study, we utilise 30 discrete weather patterns, which is a different approach to the majority of prior research in this area. Studies using fewer patterns focus more on specific regimes common to the North Atlantic such as the phase of the North Atlantic Oscillation (NAO) or broader pattern clusters such as Scandinavian Blocking or Atlantic Ridging (i.e. Falkena et al. 2020). By focussing on a smaller number of regimes, it is a simpler task to relate changes to specific large-scale drivers, to assess changes over recent decades (i.e. Marshall 2021) and project the cause of changes in the regimes into the future (i.e. Fabiano et al. 2021).

However, by using the larger number of weather patterns, we are able to investigate finer scale changes. For example, a shift to $\mathrm{NAO}+$ conditions will predominantly lead to wetter conditions over the British Isles in winter, however the exact location of the high and low pressures will determine the areas of the British Isles being exposed to the majority of the associated precipitation. For planners interested in potential changes in future winter storms, the information available from which specific weather patterns change in frequency will enable them to understand how their region is likely to be affected. This level of detail is not feasible in an analysis focusing on the large-scale regimes. Additionally, these 30 weather patterns can be distilled into a smaller set of 8 regimes (Neal et al. 2016); directly from the results that are presented here, enabling the user to gain the benefit of both the granularity from a high number of patterns and the simplicity of analysing regimes focussed on specific main circulation drivers.

In addition to providing a classification of the occurrence of meteorological conditions, the weather patterns also provide a pathway to associating specific events with particular weather conditions. By combining information on the occurrence of specific events with measures of the large-scale circulation, it is possible to assess the contribution of the meteorological conditions on an event. For example, Huang et al. (2020) utilised mortality data with EOFs of North Atlantic $500 \mathrm{hPa}$ geopotential height to assess the role of temperature on excess mortality in the UK. They then utilise this as a pathway to producing sub-seasonal and medium range forecasts of temperature related excess mortality.

The UK Climate Projections (UKCP) 2018 project has produced a range of projections for $21^{\text {st }}$ Century UK climate, including three model-based products at three spatial scales: global, regional (North Atlantic-European domain) and local (UK domain). Here, we used daily mean sea level pressure (MSLP) data from the global-scale UKCP model product and the predefined 30 Met Office weather patterns to investigate the changes in the frequency and persistence of differing circulation types. These two metrics are of greatest interest to planners due to their related temperature and precipitation impacts, for example an increase in the frequency of low pressure systems and a decrease in the persistence of these patterns could indicate an overall increase in the number of storm events and an increase in flood event risk. In combination with an updated climatology based on the ensemble, the paper outlines a methodology for understanding future changes in weather patterns over the UK through the 21 st Century and how they could be translated to climate hazards. Results focus on the end of the 21st Century (2071-2099) and includes a case study of how the weather patterns could be used in an impacts focussed analysis.

\section{Methods}

\subsection{UKCP}

UKCP Global (Met Office Hadley Centre 2018) comprises two sets of model simulations, a 15-member perturbed parameter ensemble (PPE) based on the UK Met Office Global Coupled Model and a 13-member multi-model ensemble (MME) selected from phase 5 of the Coupled Model Intercomparison Project (CMIP5-Taylor et al. 2012) ensemble. This will enable us to investigate the role of model parameter and model structural uncertainty. By utilising two emissions scenarios, we will be able to investigate the effects from emissions uncertainty on these results as well. The 15-member PPE was based on the GC3.05 configuration of the UK Met Office Global Coupled Model (Williams et al. 2017), a fully coupled version of the UK Met Office model featuring atmosphere and land (Walters et al. 2017), ocean (Storkey et al. 2017), and sea ice (Ridley et al. 2017) components. Perturbations and the process for constructing the PPE used in UKCP Global is discussed in Sexton et al. (2021) and the performance of the PPE is discussed in Yamazaki et al. (2021). The 13 CMIP5 models were selected representing 12 different modelling groups, including a previous configuration of the UK Met Office model, HadGEM2; the selection was based on work by McSweeney et al. (2015), which is summarised in Murphy et al. (2018). Details of the CMIP5 models can be found in Supplementary Table 1, 
please note that the CMIP5-13 models have been regridded onto the same $60 \mathrm{~km}$ latitude-longitude grid of the PPE- 15 . Throughout this paper we analyse the UKCP Global ensemble types (PPE and MME) in parallel, referring to the PPE15 for the perturbed parameter ensemble and the CMIP5-13 for the CMIP5 members.

All 28 simulations were run from 1st December 1899 to 30th November 2099 at N216 horizontal resolution, representative of a horizontal grid spacing of $60 \mathrm{~km}$ at the equator. The period from 1900 to 2005 represents the historical forcing phase and 2006-2099 the projection forcing phase of each simulation. The historical phase was forced with the CMIP5 timeseries of observed greenhouse gas concentrations, ozone, solar radiation, natural and anthropogenic aerosol emissions and major volcanic eruptions, used in the historical simulations contributed to CMIP5 (Taylor et al. 2012). These forcings were the same across all members of the UKCP Global ensemble, with the exception of sulphur dioxide within the PPE-15, where uncertainty in emissions was also sampled through a perturbed parameter within the aerosol component, GLOMAP-mode (Carslaw et al. 2013). Similarly, changes in land vegetation types were prescribed based on the land-use reconstructions used in CMIP5 (Hurtt et al. 2011); coverage of land ice, soil and urban areas was kept unchanged. From 2006 to 2099, climate forcing agents were applied using two Representative Concentration Pathway (RCP) scenarios (Van Vuuren et al. 2011), RCP 2.6 and RCP 8.5 (note that the sulphur dioxide parameterisation was maintained for each member of the PPE-15 in both scenarios). To represent uncertainty in carbon cycle feedbacks, each member of the PPE-15 was forced with a subtly different variant of each scenario. For RCP 2.6 this resulted in a $\mathrm{CO}_{2}$ concentration by 2100 across the ensemble ranging from 422 to 464 (note that the standard model variant was set at 422) parts per million (ppm). For RCP 8.5, this resulted in an ensemble range in $\mathrm{CO}_{2}$ concentration by 2100 ranging from 862 to 1268 (note that the standard model variant was set at 932) ppm. The CMIP5-13 members used the standard value of $\mathrm{CO}_{2}$. The distribution of the $\mathrm{CO}_{2}$ pathways used is shown in Supp. Figure 1. Weather pattern classifications were not produced for the CMIP5-13 ensemble for RCP 2.6 as MSLP data are not available from the CMIP5 database for use within UKCP Global.

\subsection{The set of $\mathbf{3 0}$ Met Office daily weather patterns}

Weather patterns are already used to forecast the likelihood of different circulation types in medium- to longrange time-scales (i.e. the one to six week period), driven by EPS predominantly used in NWP forecasting (e.g., Ferranti and Corti 2011; Neal et al. 2016). Due to the large amount of available information, the analysis of an EPS can be time consuming, therefore by using predefined weather patterns and objectively assigning each member of the NWP ensemble to its nearest weather pattern, the ensemble forecast can be translated into a group of circulation type probabilities. Each weather pattern then has its own associated climatological characteristics for a range of meteorological variables (such as temperature and precipitation) allowing a given weather pattern to be translated into expected weather impacts for a particular region. Here, we have used the set of 30 daily weather patterns which are currently used in operational medium- to longrange weather forecasting by the Met Office as described in Neal et al. (2016). The choice of 30 was based on an aim to produce a set of patterns that represented the full range of circulation types affecting the UK and surrounding European area (Neal et al. 2016). These patterns were created by using a non-hierarchical k-means clustering algorithm (Philipp et al. 2007) and applying it to the European and North Atlantic Daily to Multi-Decadal Climate Variability (EMULATE-Ansell et al. 2006) dataset. EMULATE is a gridded daily historical MSLP anomaly dataset for a North Atlantic-European domain $\left(30^{\circ} \mathrm{W}-20^{\circ} \mathrm{E} ; 35^{\circ}-70^{\circ} \mathrm{N}\right)$ at $5^{\circ}$ horizontal resolution for the period 1850-2003.

These weather patterns are gridded MSLP anomalies (Fig. 1), with lower numbered patterns occurring most often annually (around 6\% of the time) and higher numbered patterns occurring least often annually (around 1\% of the time). The lower-numbered weather patterns tend to occur more often in the summer months with the highernumbered weather patterns tending to occur more often in the winter months. Therefore, the lower-numbered weather patterns represent the more day-to-day circulation types most common on annual timescales. The higher-numbered weather patterns have some of the larger MSLP anomalies (both positive and negative) and can represent some severe weather circulation types. For example, weather pattern 30 is cyclonic with a deep negative pressure anomaly located over the Faeroe Islands, extending its influence over the British Isles-leading to very wet and windy conditions during all seasons. Another potentially severe weather example, weather pattern 27-has a positive pressure anomaly to the north of the British Isles and a negative pressure anomaly to the south-which may lead to the advection of very cold air and snow showers over eastern parts in winter. A brief description of the 30 weather patterns can be found in Table 1 .

Throughout this work, we will refer to four different time periods:

(i) Historical period-January 1900 to December 2010.

(ii) Anomaly period-January 1900 to December 1999.

(iii) Evaluation period-January 1979 to December 2010.

(iv) Analysis period-January 2071 to November 2099. 


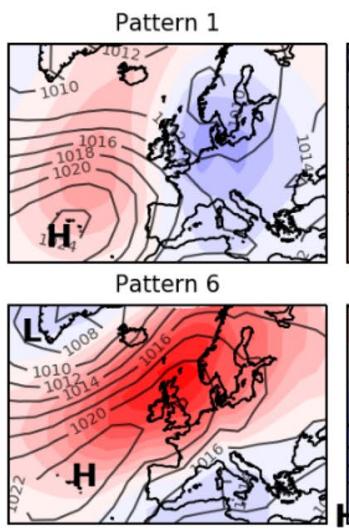

Pattern 11

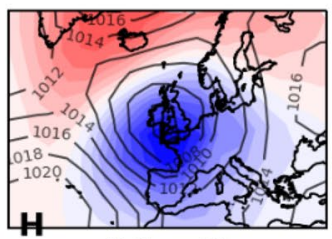

Pattern 16

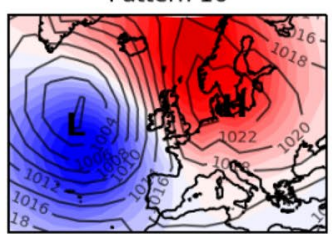

Pattern 21
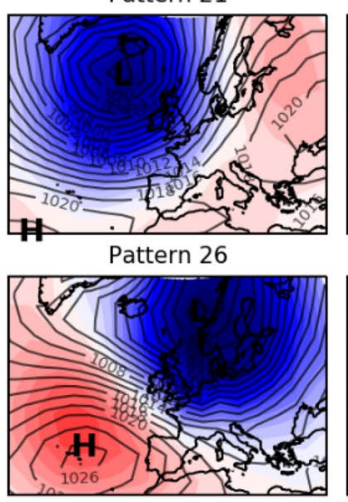

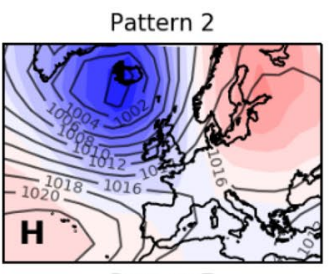

Pattern 7

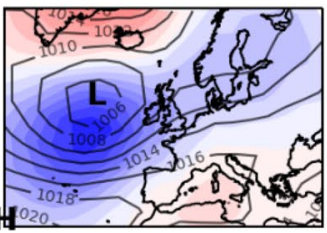

Pattern 12

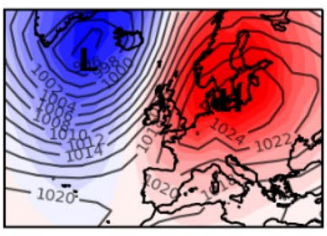

Pattern 17

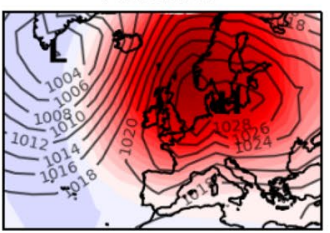

Pattern 22

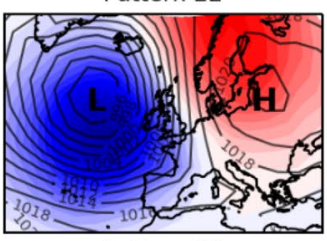

Pattern 27

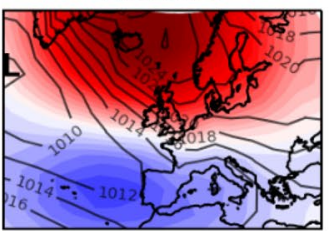

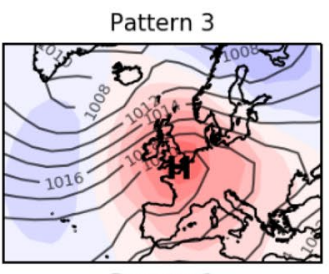

Pattern 8

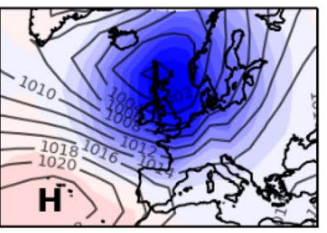

Pattern 13

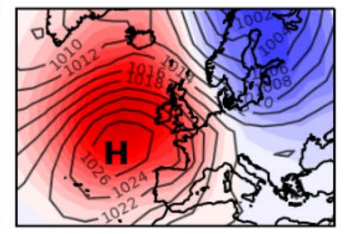

Pattern 18

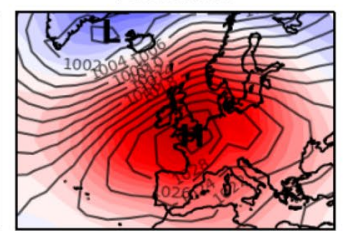

Pattern 23

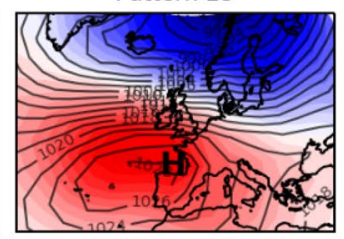

Pattern 28

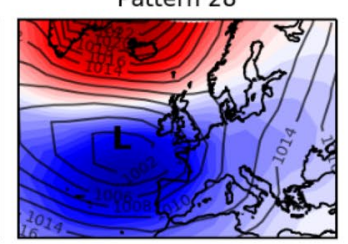

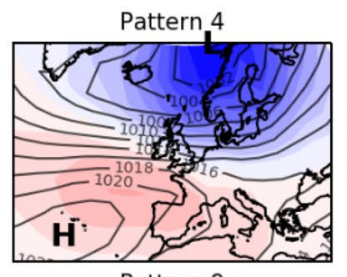

Pattern 9

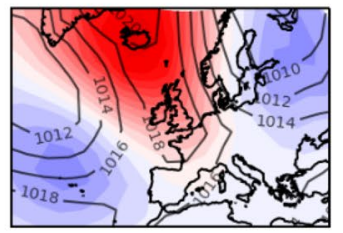

Pattern 14

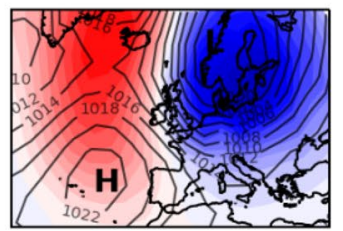

Pattern 19

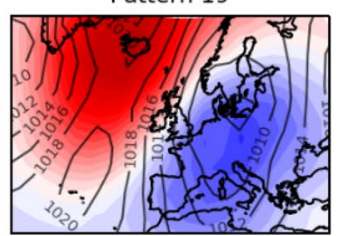

Pattern 24

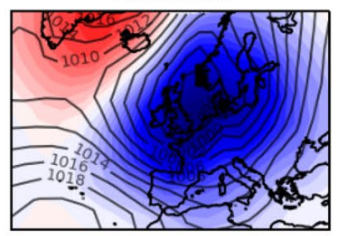

Pattern 29

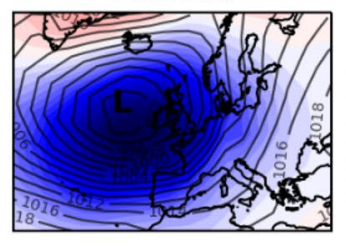

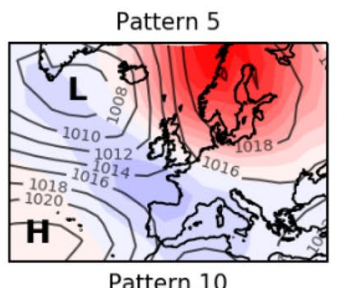

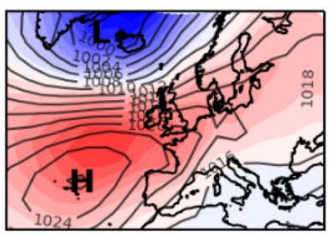

Pattern 15

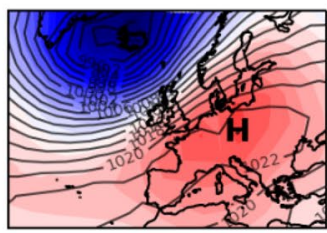

Pattern 20

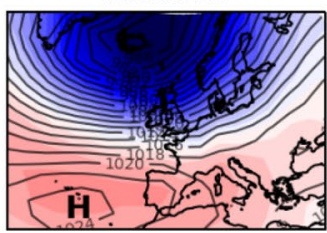

Pattern 25
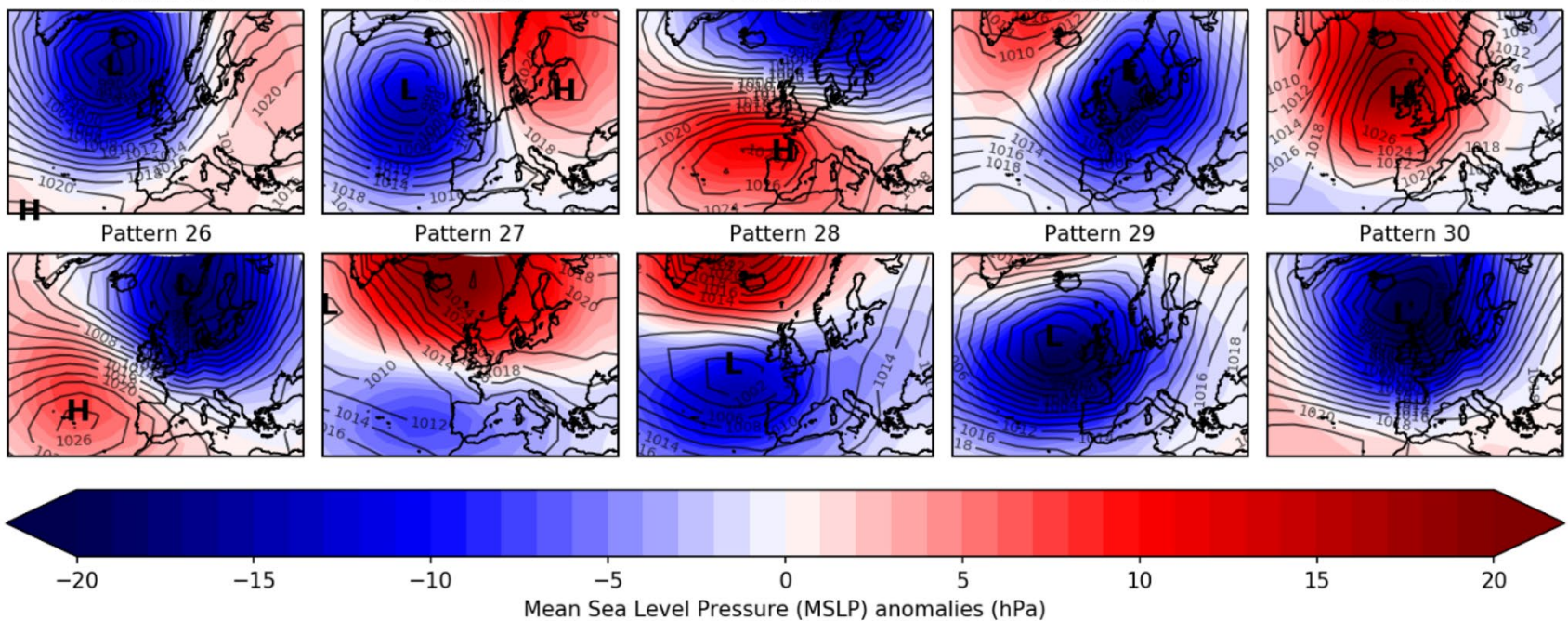

Fig. 1 Definition maps for the set of 30 predefined weather patterns from Neal et al. (2016) used in this study. Mean sea level pressure (MSLP) anomalies plotted as filled contours (hPa) and MSLP mean

We investigate the future changes in the weather patterns that we experience today, and present results based on the end-of-century changes to highlight the potential differences between emissions scenarios. We apply Neal et al.'s (2016) methodology to assign weather patterns to the UKCP Global projections. This requires, for each member, calculating the MSLP daily anomalies compared values plotted in foreground ( $2 \mathrm{hPa}$ intervals). A description of each weather pattern is given in Table 1

to the mean of the corresponding day of the year for the Anomaly Period. The MSLP anomalies are then regridded to $5^{\circ}$ latitude-longitude resolution over the weather pattern domain. Weather patterns are assigned based on their closest distance-defined as the area-weighted sum of squares difference at each grid point (Neal et al. 2016). 
Table 1 Descriptions of each of the 30 weather patterns shown in Fig. 1

\begin{tabular}{|c|c|}
\hline $\begin{array}{l}\text { Weather } \\
\text { pattern }\end{array}$ & Description \\
\hline 1 & Unbiased north-westerly \\
\hline 2 & Cyclonic south-westerly, returning Polar maritime airmass \\
\hline 3 & Anticyclonic south-westerly, ridge over Northern France \\
\hline 4 & Unbiased westerly \\
\hline 5 & Unbiased southerly, high over Scandinavia \\
\hline 6 & Anticyclonic, Azores high extension \\
\hline 7 & Cyclonic south-westerly, low west-northwest of Ireland \\
\hline 8 & Cyclonic westerly, low near Shetland \\
\hline 9 & Anticyclonic north or north-easterly, high near Iceland \\
\hline 10 & Anticyclonic west or south-westerly, slight Azores ridge \\
\hline 11 & Cyclonic, low centred over southern UK \\
\hline 12 & Anticyclonic southerly, high over Poland \\
\hline 13 & Anticyclonic north-westerly, high south west of Ireland \\
\hline 14 & $\begin{array}{l}\text { Cyclonic north or north-westerly, low near southern } \\
\text { Sweden }\end{array}$ \\
\hline 15 & $\begin{array}{l}\text { Unbiased south-westerly, very windy over north-west } \\
\text { Britain }\end{array}$ \\
\hline 16 & Anticyclonic south or south-easterly, high east of Denmark \\
\hline 17 & Anticyclonic east or south-easterly high over Denmark \\
\hline 18 & Anticyclonic south-westerly, high over northern France \\
\hline 19 & Unbiased northerly, low east of Denmark \\
\hline 20 & Cyclonic westerly, intense low near Iceland \\
\hline 21 & Cyclonic south-westerly, deep low south of Iceland \\
\hline 22 & Cyclonic southerly, low west of Ireland \\
\hline 23 & Unbiased westerly, windy in north of the UK \\
\hline 24 & Cyclonic northerly, low in North Sea \\
\hline 25 & Anticyclonic northerly, high centre in Irish Sea \\
\hline 26 & Cyclonic north-westerly, low near Norway, windy \\
\hline 27 & Anticyclonic easterly, high in Norwegian Sea \\
\hline 28 & Cyclonic south-easterly, low SW of UK \\
\hline 29 & Cyclonic south or south-westerly, deep low west of Ireland \\
\hline 30 & $\begin{array}{l}\text { Cyclonic west or south-westerly, deep low south-east of } \\
\text { Iceland }\end{array}$ \\
\hline
\end{tabular}

\subsection{Evaluating UKCP global assigned weather patterns}

We evaluate the performance of UKCP Global by comparing with the European Centre for Medium Range Weather Forecasting (ECMWF) ERA5 reanalysis (Hersbach et al. 2020) for the evaluation period (1979-2010). ERA5 is the replacement for the ERA-Interim reanalysis (Dee et al. 2011) and is available with a horizontal resolution of $31 \mathrm{~km}$ and currently a daily temporal resolution for the period 1979-present. Similarly apply the Neal et al (2016) method to assign weather patterns to the ERA5 dataset.

\subsection{Assessing changes in frequency and persistence}

In the analysis of the weather patterns generated from the UKCP Global ensemble, we assess two key metrics. The first is the frequency of each weather pattern, whereby we derive the average daily occurrence of each weather pattern on annual and seasonal timescales. The second is the persistence, a measure of the average time each weather pattern affects the UK without interruption. An additional metric is the number of events greater than or equal to four days in duration, where the number of events lasting four days or longer was assessed. The minimum of four days was chosen as this would enable the establishment and persistence of a pattern that would meet the criteria of a heatwave event in the UK (McCarthy et al. 2019); however the metric would be of use for prolonged periods of patterns associated with wet weather.

We counted the occurrence of each weather pattern in the daily data across each individual member of the PPE15 and CMIP5-13 ensembles. A percentage frequency can then be calculated based on the sum of the occurrence of each weather pattern against the total number of weather patterns assigned.

To test the statistical significance of the changes seen between the Analysis Period and Historical Period for each ensemble (PPE-15 for both RCP 2.6 and RCP 8.5, and the CMIP5-13 at RCP 8.5), we use the Pearson chi-squared test (Pearson 1900) to determine if there is a relationship between the Historical Period and the Analysis Period. The null hypothesis states that there is no association between the period and weather pattern regime (that is that the occurrence of weather patterns does not vary), and that as a result there is no evidence to support the assertion that the climate forcing has driven the changes between these two periods. If the result is statistically significant then the differences between the Analysis Period and the Historical Period are greater than the variability that would be expected and as such could be a result of the climate forcing. The chi-squared test was applied to the output from individual ensemble members in six groups based on season (winter (DJF) or summer (JJA)), ensemble (PPE-15 or CMIP5-13) and for the PPE-15 the two RCP scenarios (RCP 2.6 and RCP 8.5), as a result the threshold for significance was lowered using the Bonferroni Correction from 5 to $0.83 \%$. This is to account for the risk of increasing Type 1 errors when multiple hypothesis tests are undertaken. Chi-squared tests are sensitive if more than $20 \%$ of the cells in a table have an expected frequency that is less than 5 . All six groupings (tables) were checked for cells where the expected frequency was less than 5 and none were found.

Persistence was calculated as the total number of days with each pattern assigned to it divided by the number of occurrences of that pattern. An occurrence is each block 
of that pattern irrespective of its number of days. For example, if pattern 6 had the following durations in days for a summer period:

$1-1-2-3-4-5-4-3-2-1-1-2$,

then it has a persistence of 2.42 days ( 29 days of duration divided by 12 occurrences of the pattern). Four-day persistence (a measure of the frequency of events of a specific length) was determined as the count of the number of occurrences when a pattern lasted for four days or more divided by the number of occurrences of that pattern. In our example above, four-day persistence is 0.25 ( 3 events of four days or longer divided by the 12 occurrences of the pattern). Both measures of persistence were calculated within each individual ensemble member and then combined to form the ensemble mean. While this study uses a larger number of patterns, the focus on changes in frequency and persistence have been utilised for similar studies before (i.e. Fabiano et al. 2020; Michelangeli et al. 1995), however this is the first application of these techniques on a large number of patterns currently used within operational meteorology.

\subsection{Changes in the climatology associated with each weather pattern}

Each weather pattern is associated with a particular climatology, which will be affected by the simulated changes in the climate across the UKCP Global ensemble. These climatologies provide the expected conditions associated with each weather pattern. Here, the British Isles (land only) climatological averages for the historical period (1900-2010) and analysis period (2071-2099) were calculated for surface temperature and precipitation.

\section{Results}

\subsection{Evaluation of UKCP global with ERA5}

On annual timescales, the ERA5 weather pattern frequency of occurrence fall inside either or both the PPE-15 and CMIP5-13 ensemble ranges (Fig. 2), with the exception of pattern 13. Focussing on winter (DJF) and summer (JJA), there is again good agreement between the two ensembles from UKCP Global and the ERA5 weather pattern frequency (Fig. 3), with all but patterns 7, 17 and 27 in winter within

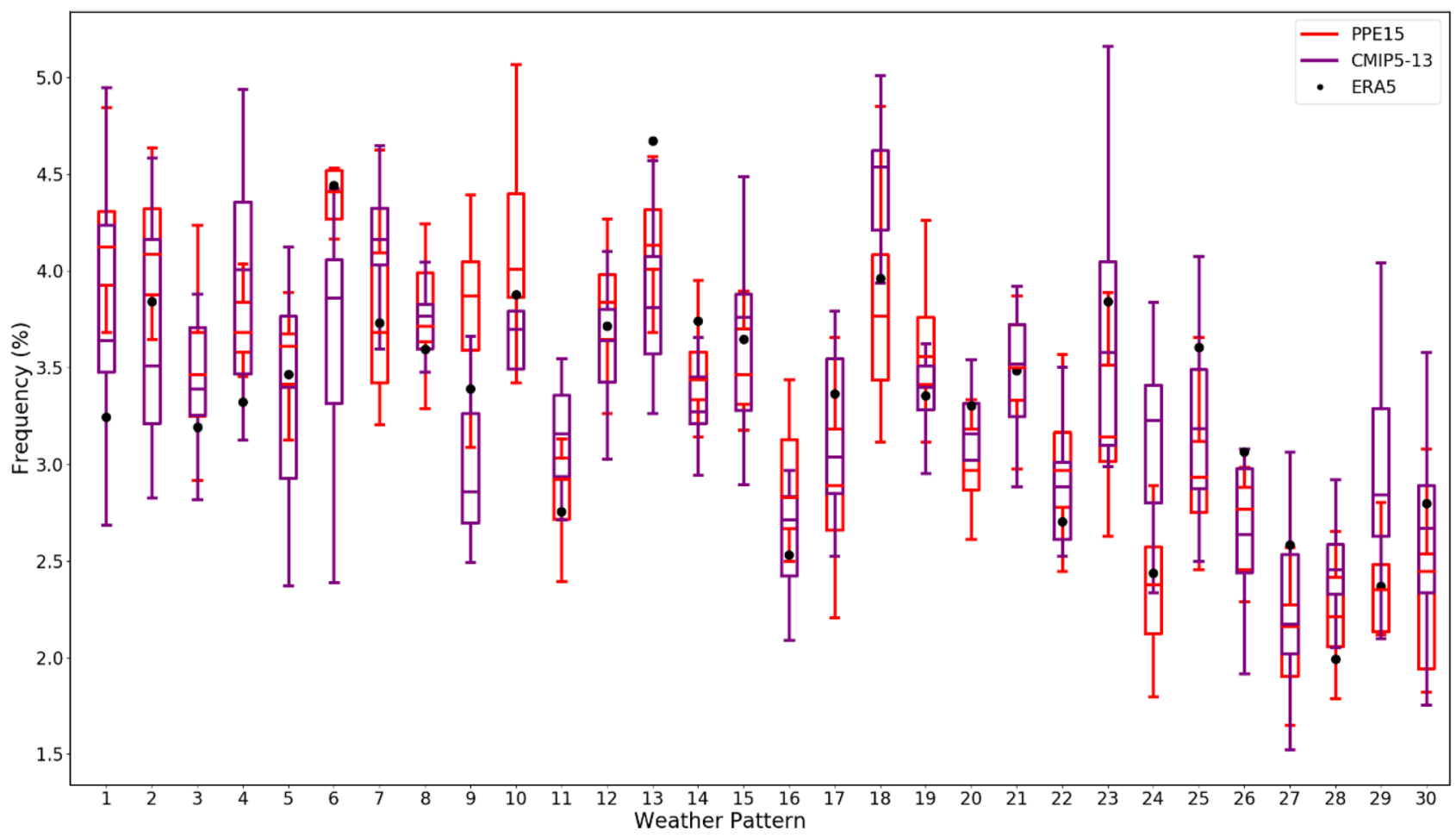

Fig. 2 Mean annual frequencies for the 30 weather patterns from the PPE-15 (red) and CMIP5-13 (purple) for the Evaluation Period in comparison to the weather pattern frequencies from the ERA5 reanalysis (black dots) for the same period. Boxes display the lower and upper quartile ranges with the median marked by a horizontal line. The whiskers extend from the box to the maximum and minimum extreme, non-outlier data points 

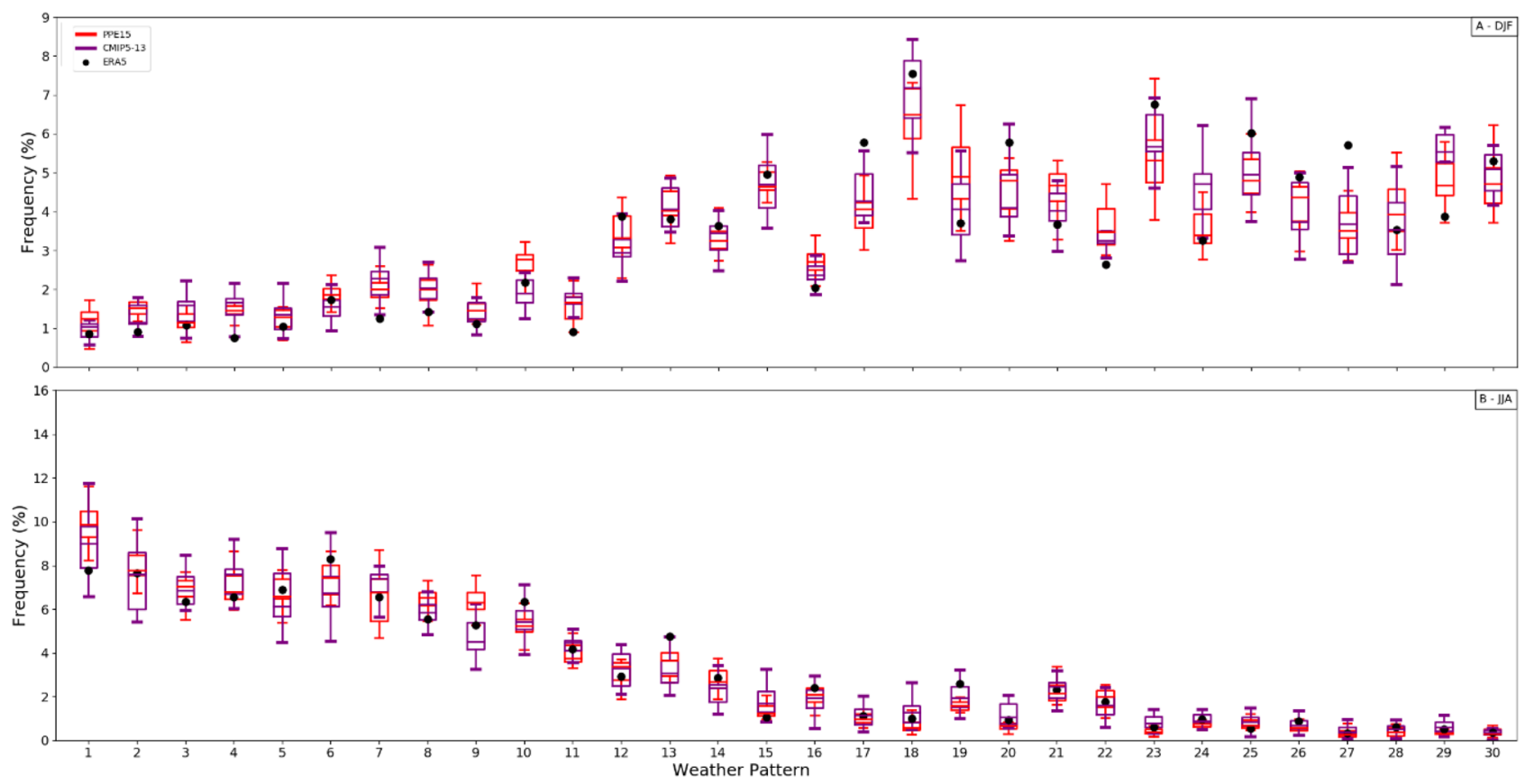

Fig. 3 Mean A winter (DJF) and B summer (JJA) seasonal frequencies for the 30 weather patterns from the PPE-15 (red) and CMIP5-13 (purple) for the evaluation period in comparison to the weather pattern frequencies from the ERA5 reanalysis (black dots) for the same

the range of either of or both the PPE-15 and CMIP5-13 ensembles. There is a similar response for the spring (MAM) and autumn (SON), with only pattern 1 in autumn falling outside the range of either of the ensembles (Supp. Fig. 2). A similar analysis was also carried out using EMULATE which showed less agreement with UKCP Global (not shown). Based on this analysis, we demonstrate that the agreement between the ensemble representation of patterns and their occurrence is respectable. This is likely due to the spatial resolution of the EMULATE dataset $\left(5^{\circ}\right.$ horizontal resolution) which is coarser than the PPE-15 and CMIP5-13 $\left(0.56^{\circ}\right.$ horizontal resolution $)$ and ERA5 $\left(0.28^{\circ}\right.$ horizontal resolution). This means that EMULATE is unable to resolve smaller low-pressure systems with deeper anomalies.

\subsection{Future changes in the frequency of weather patterns}

To assess changes into the future, the simulated weather patterns for the Analysis Period at the end of the 21st Century are compared to the weather patterns simulated for the Historical Period with a focus on the winter and summer seasons, which display the strongest signal in the distribution of the weather patterns. Each pattern represents a different climatological impact on the British Isles, so frequency changes could correspond with significant impacts in certain regions. Using a threshold of a percentage change in period. Boxes display the lower and upper quartile ranges with the median marked by a horizontal line. The whiskers extend from the box to the maximum and minimum extreme, non-outlier data points

frequency of greater than $\pm 20 \%$, nine patterns in the RCP 8.5 PPE-15 simulations $(3,4,14,15,18,20,23,26$ and 30) display an increase in winter (Fig. 4a), with a further ten showing a decrease $(2,5,11,16,17,19,22,27,28$ and 29). Eight of the nine patterns with increasing frequency have a westerly component to them with a mix of cyclonic, unbiased and anticyclonic types over the British Isles.

All nine patterns that see an increase can produce windy conditions across different regions of the British Isles. Patterns 3 and 18 are the only patterns where the British Isles (and much of the wider North Atlantic-European domain) are under the influence of the same high-pressure anomaly. The remaining patterns all have a low-pressure anomaly of varying depths centred no further south than approximately $56^{\circ} \mathrm{N}$, a distribution associated with the positive phase of the North Atlantic Oscillation (NAO), which brings milder and wetter conditions in winter to the British Isles. A separate analysis of the UKCP Global simulations indicated an overall tendency towards the positive phase of the NAO through the course of the 21st Century (McSweene and Yamazaki 2020).

In contrast, the decreasing patterns are less likely to have a westerly component to them with patterns split between those with northerly or southerly components. Of the eight patterns that show a decrease, half are patterns whose likely airmass over the UK would be Polar continental $(5,16,27)$ or Arctic maritime (19) which during 


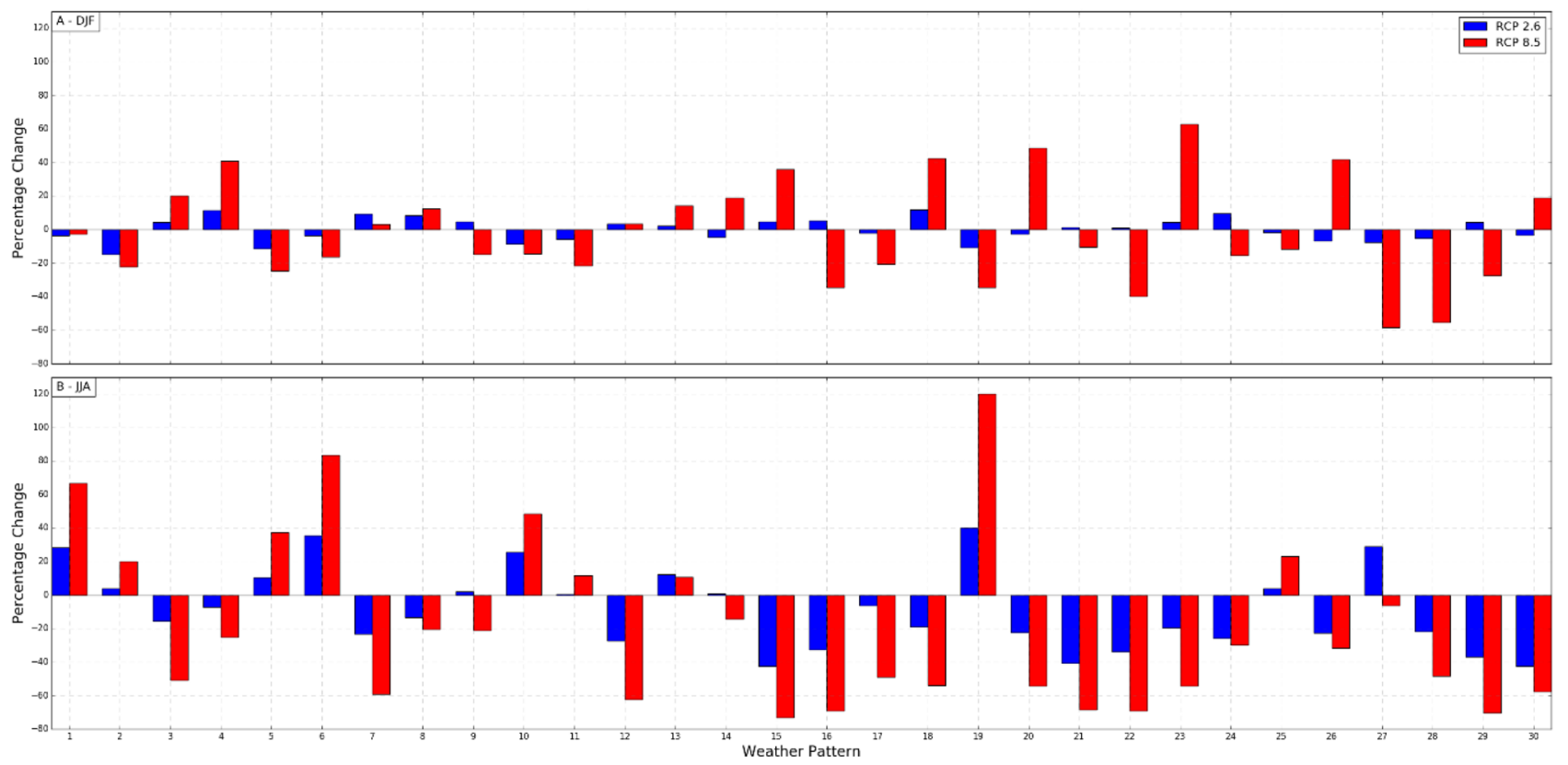

Fig. 4 Ensemble mean percentage change in frequencies of the 30 weather patterns between the analysis period and the historical period for $\mathbf{A}$ winter (DJF) and B summer (JJA) for the PPE-15 simulations for RCP 2.6 (blue) and RCP 8.5 (red)

winter generally bring cooler conditions. Of the remaining, 28 is finely balanced with a continental flow and 11, 22 , and 29 are likely to bring warmer conditions.

For the summer (Fig. 4b), seven weather patterns in the RCP 8.5 PPE-15 simulations (1, 2, 5, 6, 10, 19 and 25) display an increase (with patterns 1,6 and 19 displaying greater than $50 \%$ increase) in frequency while eighteen weather patterns $(3,4,7,9,12,15,16,17,18,20,21$, $22,23,24,26,28,29$ and 30) show a decrease. Six of the seven increases represent anti-cyclonic or unbiased patterns and typically represent relatively settled and dry types for much of the British Isles, whilst nine of the eighteen decreasing patterns represent cyclonic patterns; the overall pattern is a noticeable decrease in the frequency of the higher numbered weather patterns associated with stormy and wet conditions, which climatologically are the rarer patterns in the summer already (Fig. 3b).

There are noticeable differences in the frequencies for the PPE-15 RCP 2.6 compared to RCP 8.5 In both winter (Fig. 4a) and summer (Fig. 4b), the RCP 2.6 simulations display a similar distribution of changes in frequency; however the magnitude of these changes is smaller than seen in the RCP 8.5 simulations. A noticeable outlier is the response in weather pattern 27 during summer, which has an increase of greater than 20\% in the RCP 2.6 simulation, compared to negligible change in RCP 8.5 (Fig. 4b). This indicates that under lower emissions scenarios, weather patterns are projected to be similar to those experienced in the present day.
For both seasons, the response of the CMIP5-13 simulations is broadly the same as that shown in the PPE-15 simulations; however the CMIP5-13 results have a smaller magnitude (Supp. Fig. 3). When assessing the individual ensemble members of both the PPE-15 and the CMIP5-13, there is a much broader spread in the ensemble results for the CMIP5-13, as displayed in Figs. 2 and 3 (and Supp. Fig. 4, $5)$. This is consistent with analysis of the UKCP modelling outputs (c.f. Fig. 5.1, Kendon et al. 2019). The PPE-15 and CMIP5-13 represent two different methodologies, one based on perturbing a single climate model and the other an ensemble based on a range of different climate models (an "ensemble of opportunity"), for constructing ensembles of climate models and these differences can explain the difference in ensemble spread. The broad agreement between the PPE-15 and CMIP5-13 indicates that the projected changes in weather pattern frequency are robust across different methodologies for creating ensembles, however there are clearly a spread of responses across the ensemble members (Supp. Fig. 4). For weather patterns with mean changes that are similar between the RCP 8.5 PPE-15 and the CMIP5-13 simulations (Supp. Fig. 3) there is a lot of overlap between the individual members between the two ensembles. The main differences between the ensemble means correspond with the greatest differences between the individual ensemble members. For example, pattern 19 in the summer, displays a strong disagreement between the two ensembles (Supp. Fig. 4b), only four members of the CMIP5-13 overlap with the PPE-15 members. Finally, the spread in the 
ensembles highlights the impact of outlier members in either of the ensembles. For example, pattern 27 in the summer shows good agreement between the two ensembles aside from one member of the CMIP5-13 which displays an exceptionally large change, and as such weather pattern 27 shows a different mean response between the two ensembles (Supp. Fig. 3). Comparing the results between the ensemble means for the RCP 8.5 PPE-15 and RCP 2.6 PPE-15 simulations (Fig. 4) with their ensemble member spreads (Supp. Fig. 5), there is a similar range in spread across the two forcing scenarios, with the mean results well represented in the ensemble spreads. A chi-squared test was applied to each of the datasets shown in Fig. 4 and Supplementary Fig. 3, with the six datasets all returning a significant difference between the analysis period and the historical period.

\subsection{Changes in the persistence and climatology of the weather patterns}

In winter for RCP 8.5, persistence decreases in the PPE15 simulations for all weather patterns during the analysis period (Fig. 5a), with the exception of weather patterns 4 , 18 and 23 , which show modest increases $(<5 \%$ percentage change). There is a broadly similar result in the RCP 2.6 simulations, in addition to Patterns 4 and 18 , there are modest increases $(<5 \%)$ in patterns $6,7,8,10$ and 11 , while pattern 23 (unbiased or anticyclonic westerly type) shows a decrease rather than increase in RCP 2.6. Focussing on four-day persistence, most patterns display a decrease, however patterns 4 and 23 (both westerly types for RCP 8.5), patterns 3, 7 and 15 (all south-westerly types for RCP 2.6) and patterns 11 and 18 (contrasting low and high pressure anomaly types respectively for both scenarios) indicate an increase (Supp. Fig. 6a). The CMIP513 compares favourably to the PPE-15 in winter (Supp. Fig. 7a) with only patterns 2, 4, 19, 23 and 27 showing different responses.

By contrast, for the summer, nine mostly anticyclonic or unbiased weather patterns display an increase in persistence $(1,2,5,6,10,13,14,19$, and 25) in the RCP 8.5 simulations during the analysis period (Fig. 5b). This is replicated when looking at the RCP 2.6 scenario, with the exception of pattern 25 (high pressure anomaly over the British Isles), which shows a modest decrease. As noted in the winter, the fourday persistence response for both sets of simulations replicate the average persistence response (Supp. Fig. 6b). One difference is the RCP 2.6 response is pattern 28 (cyclonic south-easterly with a low pressure anomaly centred southwest of Ireland) which shows a large increase in four-day persistence compared to the Historical Period. For both persistence metrics, the changes in the RCP 2.6 simulations is smaller than in the RCP 8.5 simulations across most weather patterns, as noted in the changes in frequency. Similarly, the response in the CMIP5-13 members in comparison to the PPE-15 (Supp. Fig. 7b) is smaller, however a modest increase in persistence ( 5\%) is simulated by the CMIP5-13 for patterns 26, 27, 28 and 30 during summer, whereas the PPE-15 shows $\sim 5 \%$ decreases for the same patterns.

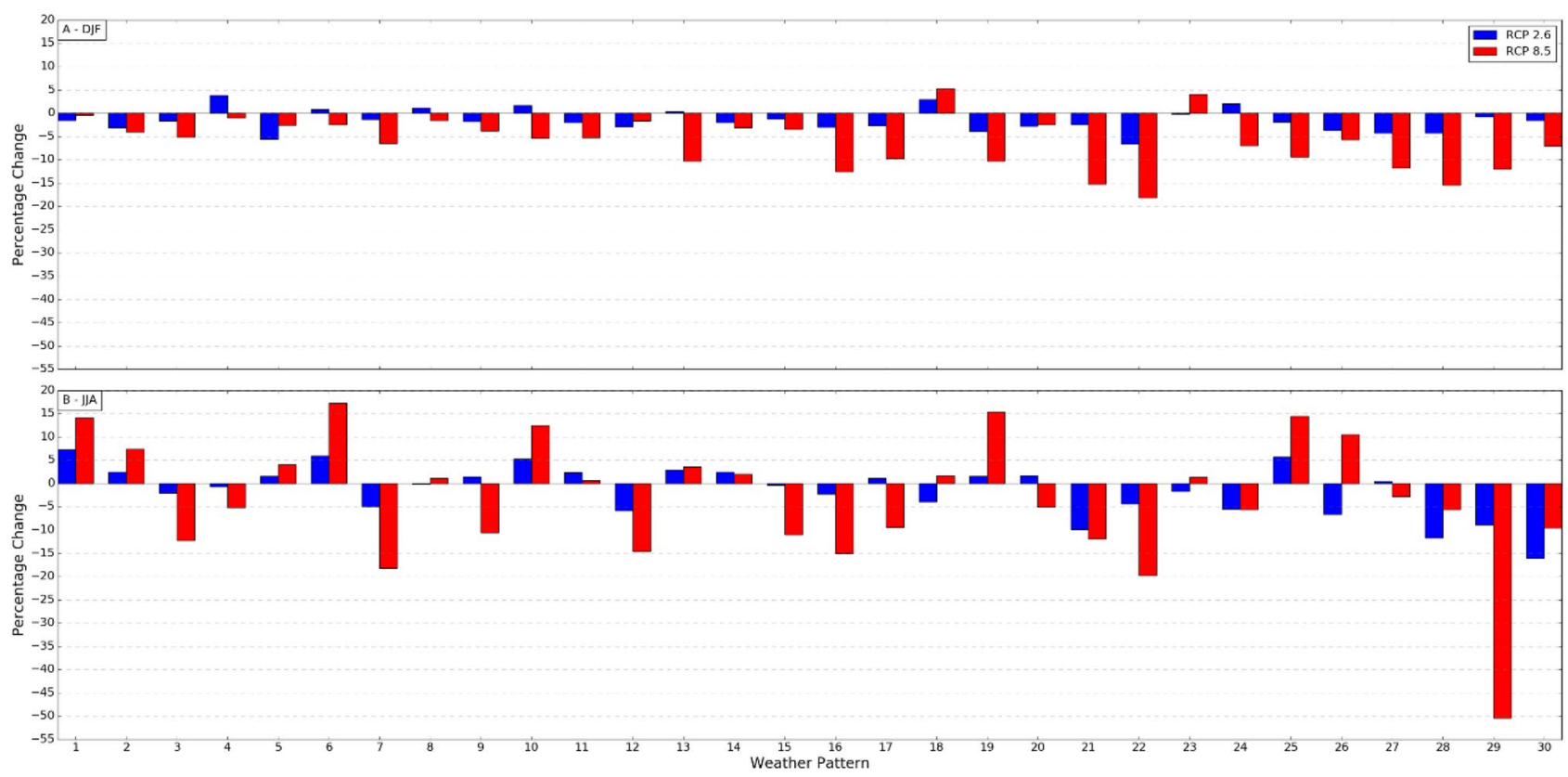

Fig. 5 Ensemble mean percentage change in average persistence of the 30 weather patterns between the analysis period and the historical period for $\mathbf{A}$ winter (DJF) and $\mathbf{B}$ summer (JJA) for the PPE-15 simulations for RCP 2.6 (blue) and RCP 8.5 (red) 
In addition to the weather pattern frequency and persistence metrics; each weather pattern has an associated climatology, with values shown here for the British Isles. The climatology associated with each weather pattern provides the thermodynamic component of future changes in UK climate, which complements the analysis of the changes in the dynamical changes provided by the weather patterns. Consistent with the headline findings of UKCP (Lowe et al. 2018), for the Analysis Period temperatures associated with all the weather patterns are projected to increase in both the summer and winter relative to the Historical Period, with larger projected temperature increases in the summer (Table 2). For precipitation, the UKCP headline findings indicate that winters are projected to become wetter and summers are projected to become drier (Lowe et al. 2018). The seasonal pattern response is that all but five patterns (13, $14,19,25$ and 26) display an increase in winter precipitation, while all but pattern 18 and 27 display a decrease in precipitation during the summer (Table 2). As simulated by the PPE-15 under RCP 8.5, the climatologies associated with each weather pattern for the CMIP5-13 are consistent with the UKCP headline findings, with the exception of summer precipitation for weather patterns 15 and 29, which show a slight increase and no change respectively (Supp. Table 2). Consistent with other results the RCP2.6 PPE-15 ensemble displays a similar sign of the change for each weather pattern, however the magnitude of the change is smaller than observed in the RCP 8.5 equivalent (Supp. Table 3).

\section{Discussion}

The preceding sections have introduced and assessed changes in the frequency and persistence of weather patterns over the North Atlantic-European domain by the end of the $21^{\text {st }}$ Century, and the changes in their associated climatologies over the British Isles. Now we explore how these results fit into the broader context of future

Table 2 Climatology associated with each weather pattern for the historical period and the anomaly between the analysis period and the historical period for winter and summer precipitation and temperature for the PPE-15 under the RCP 8.5 Scenario

\begin{tabular}{|c|c|c|c|c|c|c|c|c|}
\hline \multirow[b]{2}{*}{ Pattern } & \multicolumn{2}{|c|}{$\begin{array}{c}\text { Winter Precipitation } \\
\text { (mm/day) }\end{array}$} & \multicolumn{2}{|c|}{ Winter Temperature $\left({ }^{\circ} \mathrm{C}\right)$} & \multicolumn{2}{|c|}{$\begin{array}{c}\text { Summer Precipitation } \\
\text { (mm/day) }\end{array}$} & \multicolumn{2}{|c|}{ Summer Temperature $\left({ }^{\circ} \mathrm{C}\right)$} \\
\hline & $\begin{array}{l}\text { Historical } \\
\text { Period }\end{array}$ & $\begin{array}{l}\text { Analysis } \\
\text { Period }\end{array}$ & $\begin{array}{l}\text { Historical } \\
\text { Period }\end{array}$ & $\begin{array}{l}\text { Analysis } \\
\text { Period }\end{array}$ & $\begin{array}{l}\text { Historical } \\
\text { Period }\end{array}$ & $\begin{array}{l}\text { Analysis } \\
\text { Period }\end{array}$ & $\begin{array}{c}\text { Historical } \\
\text { Period }\end{array}$ & $\begin{array}{l}\text { Analysis } \\
\text { Period }\end{array}$ \\
\hline 1 & 3.37 & 0.29 & 2.31 & 3.39 & 2.78 & -0.98 & 13.30 & 6.60 \\
\hline 2 & 4.62 & 1.59 & 3.40 & 3.99 & 2.82 & -1.20 & 15.03 & 6.39 \\
\hline 3 & 3.30 & 1.26 & 5.22 & 2.66 & 1.33 & -0.56 & 15.07 & 5.88 \\
\hline 4 & 4.75 & 0.62 & 5.21 & 2.78 & 2.37 & -0.74 & 13.83 & 5.26 \\
\hline 5 & 2.99 & 1.43 & 1.36 & 4.63 & 2.28 & -1.28 & 15.47 & 7.02 \\
\hline 6 & 1.63 & 0.49 & 0.81 & 4.29 & 0.94 & -0.44 & 14.52 & 6.27 \\
\hline 7 & 4.94 & 1.37 & 4.50 & 3.02 & 3.26 & -0.96 & 14.27 & 5.96 \\
\hline 8 & 5.31 & 1.13 & 3.99 & 3.30 & 4.55 & -0.90 & 13.38 & 5.74 \\
\hline 9 & 2.61 & 0.07 & 2.04 & 3.28 & 1.16 & -0.39 & 13.84 & 6.25 \\
\hline 10 & 3.31 & 0.71 & 2.73 & 3.81 & 1.43 & $\begin{array}{l}-0.59 \\
\end{array}$ & 14.31 & 5.71 \\
\hline 11 & 3.98 & 1.88 & 1.78 & 4.58 & 5.01 & -1.33 & 13.70 & 7.01 \\
\hline 12 & 2.93 & 1.09 & 3.67 & 3.84 & 0.99 & -0.57 & 15.99 & 5.63 \\
\hline 13 & 2.67 & -0.23 & 3.73 & 2.76 & 1.22 & -0.24 & 12.92 & 4.39 \\
\hline 14 & 3.47 & -0.59 & 2.49 & 3.30 & 2.45 & -0.51 & 11.79 & 5.22 \\
\hline 15 & 4.51 & 1.33 & 5.71 & 3.43 & 2.05 & -0.18 & 14.97 & 4.02 \\
\hline 16 & 2.25 & 0.74 & 1.88 & 4.10 & 0.97 & -0.50 & 15.58 & 6.19 \\
\hline 17 & 1.22 & 0.25 & 1.26 & 4.09 & 0.41 & -0.29 & 15.36 & 4.65 \\
\hline 18 & 1.95 & 0.44 & 4.20 & 3.27 & 0.57 & 0.08 & 14.75 & 3.39 \\
\hline 19 & 2.41 & -0.07 & -0.16 & 4.51 & 2.45 & -0.73 & 12.02 & 6.33 \\
\hline 20 & 6.55 & 1.11 & 6.02 & 3.07 & 3.38 & -0.62 & 13.66 & 3.96 \\
\hline 21 & 6.51 & 2.19 & 5.76 & 3.23 & 4.35 & -0.83 & 14.52 & 5.49 \\
\hline 22 & 4.56 & 1.78 & 3.89 & 4.03 & 2.99 & -0.94 & 15.61 & 6.10 \\
\hline 23 & 4.25 & 0.30 & 6.12 & 2.62 & 1.81 & -0.31 & 13.09 & 2.82 \\
\hline 24 & 3.81 & 0.43 & 1.47 & 4.05 & 5.14 & -0.53 & 12.14 & 5.85 \\
\hline 25 & 1.30 & -0.12 & 1.53 & 3.41 & 0.55 & -0.22 & 13.28 & 4.69 \\
\hline 26 & 5.00 & -0.42 & 4.32 & 2.84 & 3.11 & -0.37 & 11.94 & 4.03 \\
\hline 27 & 1.70 & 0.26 & -0.46 & 4.53 & 0.76 & 0.03 & 14.32 & 5.83 \\
\hline 28 & 3.47 & 0.90 & 0.07 & 4.74 & 3.07 & -1.57 & 13.07 & 6.82 \\
\hline 29 & 6.01 & 1.49 & 3.91 & 3.47 & 6.25 & -0.19 & 13.51 & 5.79 \\
\hline 30 & 6.72 & 1.14 & 4.78 & 3.01 & 5.76 & $\begin{array}{l}-0.92 \\
\end{array}$ & 12.60 & 5.35 \\
\hline
\end{tabular}

Colours highlight the sign of the anomaly; for precipitation: green represents an increase, and brown represents a decrease. For temperature: red represents an increase and blue represents a decrease 
projections, especially from within UKCP, and how they provide a narrative of future changes in the weather of the British Isles. The following section will demonstrate the application of this work through a brief case study.

During winter there is a clear signal towards an increase in frequency of stormy westerly types (e.g. 20, 23, 26 and 30 ), in combination with a clear signal towards a decrease in patterns that have high pressure anomalies located over Scandinavia or Greenland (e.g. 5, 11, 16, 17, 19, 22, 24, 27 and 28) for the UKCP Global ensembles (Fig. 4a, Supp. Fig. 3a). These changes are observed across the different ensembles and forcing scenarios. The changes in weather pattern persistence indicate a reduction in the persistence of all but a couple of patterns during winter (Fig. 5a, Supp. Fig. 7a). For weather patterns with increasing frequency and decreasing persistence, this indicates that there are a greater number of individual events, which for patterns such as the stormy westerly types, could indicate more mobile systems passing over the UK. Climatologically, this would suggest a shift to conditions associated with the positive phase of the NAO (McSweene and Yamazaki 2020), a more active Atlantic jet stream (McSweeney and Bett 2020), and a resulting increase in very wet conditions and mild temperatures with fewer cold air outbreaks and snowy conditions in the winter.

Future changes in the jet stream and the drivers of this change have been a widely debated topic, due to its importance for European climate (Zappa and Shepherd 2017). Results based on the analysis of the weather patterns and the analysis of the UKCP Global products are consistent with previous studies focussing on these changes in the NAO or jet stream. For example, across CMIP3, CMIP5 and CMIP6; Harvey et al. (2020) found a trend in future projections for a strengthening of the North Atlantic jet, with particular impacts for the British Isles as the northern and southern flanks of the jet weakened, focussing the changes over the central jet. This result, built on a CMIP5-focussed study, suggested the jet was "squeezed" towards central positions through competing northern and southern influences (Peings et al. 2018). Similarly, the shift towards conditions associated with $\mathrm{NAO}+$ conditions is also represented across CMIP3, CMIP5 and CMIP6, with decreases in winter blocking projected across these ensembles (Davini and D'Andrea 2020); results also supported by analysis from Fabiano et al. (2021).

The changes in climatology associated with the weather patterns (Table 2, Supp. Tables 2, 3), indicate that all weather patterns simulate an increase in temperature and precipitation. The projection, both dynamically through the weather patterns and thermodynamically through the changes in associated climatology, of warmer and wetter winter conditions is consistent with the UKCP headline findings (Lowe et al. 2018).
During summer there is a clear signal towards an increase in frequency of anticyclonic types (e.g. 6 and 25), in addition to a clear signal towards a reduction in frequency of cyclonic stormy types (e.g. 7, 21, 22, 28, and 29), indicating a decrease in the mobile patterns that are associated with strong anomaly pressure gradients (Fig. 4b, Supp. Fig. 3b). Changes in persistence are more mixed in the summer, but certain anti-cyclonic patterns (e.g. 6) display an increase in persistence (Fig. 5b, Supp. Fig. 7b), that in conjunction with increased frequency could indicate a greater duration of blocking events in summer. The reduced frequency of cyclonic stormy patterns is linked to a reduction in their persistence, indicating that there are fewer and shorter periods of these patterns. Climatologically this would suggest a move towards warmer, drier summers with few large-scale frontal rainfall events during the summer. As with the winter, the projected climatology results (Table 2) are consistent with assertions based on the changes in weather pattern frequency. The decrease in these frontal systems could increase the likelihood of drought conditions in the UK. A climatological analysis comparing these weather patterns with precipitation data between 1931 and 2015 (Richardson et al., 2017 ) indicated that six patterns were associated with summer drought conditions $(6,9,10,12,17$ and 25); three $(6,10$ and 25) display increase in frequency in the Analysis Period in this study (Fig. 4b; Supp. Fig. 3b). This combination of changes may also see poor air quality over the UK as the flow is less likely to be off the North Atlantic, with either southerly continental air or stagnant air common over the UK (e.g. Fenech et al. 2019). As noted for the winter, the projected dynamic and thermodynamic changes here are consistent with the UKCP headline findings for summer of warmer, drier conditions (Lowe et al. 2018).

Methodologically, we chose to focus on the existing set of 30 Weather Patterns, allowing an assessment of changes in weather patterns in the future compared to recent experience. This choice enables better comparison between the future and our current experience of weather associated with these patterns, as these patterns are used currently in operational weather forecasting. However, the limitation of this choice is that we are unaware of any changes to the weather patterns. It is possible that the future would see alternative weather patterns emerging, with differing impacts on the large-scale circulation affecting the British Isles. Our analysis here does not interrogate any changes in the distribution of the pressure patterns, only assigning them to the pre-existing weather patterns based on historical data. 


\section{Case study of weather patterns application: winter storms}

Changes in weather patterns can be used to investigate impacts on the British Isles over the course of the 21 st Century. Previous studies have utilised the Met Office weather patterns as a tool for both retrospective analysis (i.e. Knight et al. 2017), and to indicate coastal flooding impacts (Neal et al. 2018). They have also been used to assess the future plausibility of extreme weather events such as the unusually warm February 2019 over the UK (Kendon et al. 2020). We build on these works by highlighting the additional information that weather patterns can potentially provide for winter storm events.

In the UK, winter storms can cause fluvial flooding with high economic impacts e.g. the wettest winter on record in the UK in 2013/2014 was estimated to have had a $£ 1.3$ billion economic impact (DEFRA 2016). Based on climatological analysis of precipitation and temperature, the majority of patterns in the PPE-15 and CMIP5-13 are wetter in winter in the Analysis Period compared to the Historical Period (Table 2; Supp. Table 2). However there is a range of changes in the magnitude of precipitation across the weather patterns, therefore the analysis focussed on the weather patterns identified through expert elicitation as being meteorologically most likely to be related to winter fluvial flooding: 2, 4, 7, 8, 11, 15, 20, 21, 22, 23, 24, 26, 29 and 30 , based on their synoptic conditions and associated climatologies. Of these patterns, eight $(4,7,8,15,20,23$, 26 and 30) show an increase in frequency in the PPE-15 during the analysis period (Fig. 4a) under the RCP 8.5 scenario, with an average percentage change of $45 \%$ for the analysis period compared to the historical period. For the CMIP5-13, six of these patterns $(4,7,15,20,23$ and 26) show an increase in frequency for the analysis period, with an average percentage change of $25 \%$ compared to the historical period.

Comparing the historical and analysis periods occurrences of these weather patterns across the PPE-15, the median occurrences per winter is higher for the analysis period than in the historical period, indicating a projected increase in the frequency of these conditions in winter in the analysis period (Fig. 6a). For the CMIP5-13, the same result is observed for the six patterns which display an increased frequency. The two patterns for which the frequency decreases in the CMIP5-13 but not the PPE-15 (patterns 8 and 30) have a median value for the analysis period smaller than the historical period (Fig. 6b). For the subset of results presented here, we have re-produced the chi-squared test undertaken in Sect. 3.2 but applying it only to the sub-selected members used in the case study. The differences between the analysis and historical periods
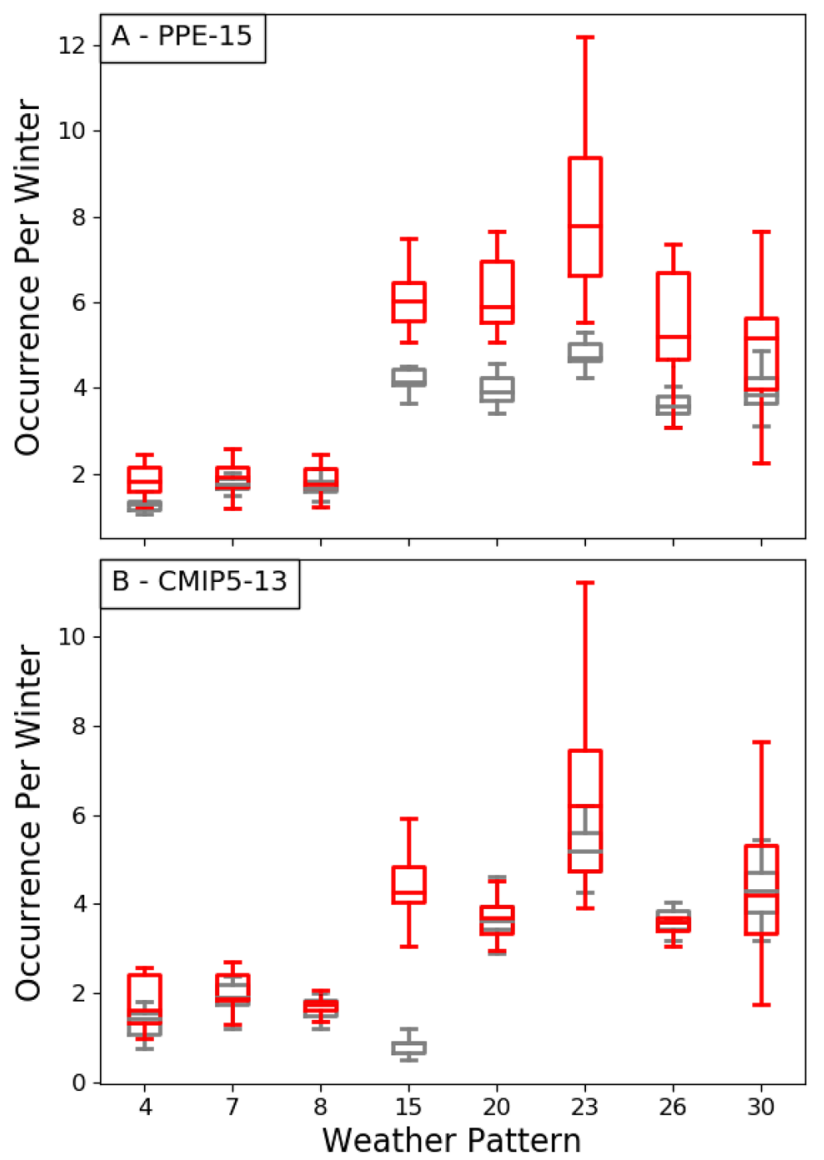

Fig. 6 Average frequency [days per winter (DJF)] across the A PPE15 and B CMIP5-13 with which weather patterns 4, 7, 8, 15, 20, 23, 26 or 30 occur for the historical period (grey) and the analysis period (red), for RCP 8.5. Boxes display the lower and upper quartile ranges with the median marked by a horizontal line. The whiskers extend from the box to the maximum and minimum extreme, non-outlier data points.

for this sub-selection of eight weather patterns was significant in both ensembles.

In addition, there is greater ensemble variability for both the PPE-15 and CMIP5-13 in all eight patterns in the analysis period compared to the historical period (Fig. 6). This variability exhibits itself in different ways, with some weather patterns having an ensemble spread in the analysis period which overlaps with much of the spread of the ensemble in the historical period. For other weather patterns, the value for the analysis period is larger than any value from the historical period. It should be noted that there is greater overlap between analysis and historical periods in the CMIP5-13 than in the PPE-15. These results would indicate that while there is a shift under an RCP 8.5 scenario for more days with impactful winter storm conditions, there is also a far greater variability in the occurrence. This is potentially a feature of the way the different models within the CMIP5-13 ensemble respond 
to changes in forcing (i.e. Peings et al. 2016; Zappa and Shepherd 2017).

\section{Conclusions}

In this paper we have combined the most recent UK Climate Projections (UKCP) with the 30 static Met Office weather patterns, with a view to understand the dynamical changes linked to large-scale circulation over the British Isles. Comparing the Analysis Period (2071-2099) to a Historical Period (1900-2010), the results indicated that in winter there is a projected increase in cyclonic and westerly types. This results in a projected reduction in more anticyclonic settled types. This indicates a shift towards warmer, wetter winters. For the summer, the results indicated an increase in settled, predominantly dry anticyclonic types at the expense of several wet and windy types. This indicates a shift towards warmer, drier summers. For both summer and winter, these results are consistent with the UKCP headline findings (Lowe et al. 2018).

The persistence of the weather patterns was also investigated and it broadly decreases in the Analysis Period compared to the Historical Period. Coupled with increased occurrence of events, this indicates that there is an increase in individual events, such as storm events, particularly in the winter. For both the frequency of occurrence and the persistence, the results were similar with respect to the type of change across both the RCP 2.6 and RCP 8.5 scenarios, however in the RCP 2.6 scenario, the magnitude of the change was smaller, indicating a smaller shift away from the Historical Period frequency and persistence of weather patterns in summer and winter.

Each weather pattern is associated with a climatology and changes in this climatology were also assessed. For the RCP 8.5 scenario, the climatology results were entirely consistent with the UKCP headline findings, with all the weather patterns warmer in both summer and winter in the Analysis Period, compared to the Historical Period. For precipitation, the majority of patterns displayed an increase in precipitation in winter and a decrease in the summer, again consistent with the UKCP headline findings. For the RCP 2.6 scenario, climatological results were consistent with, but a smaller magnitude of change than the RCP 8.5 scenario.

Finally, while this paper was focussed on the methodology for generating an assessment of future changes in weather pattern frequency, persistence and climatology, we also provided a brief example case study for applying these results. Focussing on winter storm patterns, associated with high precipitation totals, the results indicated that while the median frequency increased in all eight selected patterns (six patterns for the CMIP5-13), there was an increase in the ensemble spread, with some members displaying a decrease or no change in the average occurrence of each pattern. Therefore, while the number of impactful winter weather patterns is projected to increase in the future, there was also an increase in the uncertainty with potentially greater year-on-year variability based on the ensemble ranges.

The use of the Met Office weather patterns with climate projections such as UKCP offers a range of future research possibilities. Potential uses include investigating the likelihood of future weather events (e.g. Kendon et al. 2020), producing climate storylines (e.g. Shepherd et al. 2018) or climate risk narratives (e.g. Jack et al. 2020). All of these could be of use to planners in a range of sectors. For example, by combining the weather patterns observed for specific past events (such as the methodology used in Huang et al. (2020), it would be possible to extrapolate the changes in likelihood of these events in the future using the weather patterns derived from UKCP. This would enable planners to assess whether particular events are more or less likely in the future, and by utilising the projected changes in climatology for each pattern assess changes in the impact of these events. There is also a need to further understand the drivers of the changes seen in these results, for example is the increase in a weather pattern's frequency compensated for by a decrease in another particular pattern (a subtle shift in the sea level pressure anomalies) or does it represent a step change in the types of weather patterns occurring (a larger shift in the sea level pressure anomalies)? Similarly, there is an interest in understanding the role of inter-annual variability (in addition to the inter-seasonal variability discussed in this work) and the effect this could have on impacts based work.

Supplementary Information The online version contains supplementary material available at https://doi.org/10.1007/s00382-021-06031-0.

Acknowledgements The UKCP Global model data used in this study is all available from the Centre for Environmental Data Analysis http:// data.ceda.ac.uk/badc/ukcp18/data, cited in this study as Met Office Hadley Centre (2018). Note that for UKCP Global data set, model numbers 1-15 represent the UKCP Global PPE-15 and numbers 16-28 the UKCP Global CMIP5-13. Supplementary Table 1 outlines which number refers to which CMIP5 model name. The weather patterns for the UKCP Global ensemble are also available from the Centre for Environmental Data Analysis at the link above. JOP and FF were funded via the Met Office Hadley Centre Climate Programme, by the Department of Business, Energy and Industrial Strategy (BEIS) and the Department for Environment, Food and Rural Affairs (Defra). The participation of HMH, EJP and AR in this work was funded by the UK Climate Resilience programme, which is supported by the UKRI Strategic Priorities Fund. The programme is co-delivered by the Met Office and NERC on behalf of UKRI partners AHRC, EPSRC, ESRC. $\mathrm{KB}$ and $\mathrm{RN}$ were funded by Met Office science. The authors would like to thank the rest of the UKCP Team, particularly those responsible for running the climate models, and Met Office colleagues Dan Bernie, Carol McSweeney, David Sexton \& Hazel Thornton for their helpful discussions in formulating this work. We thank the two anonymous reviewers whose insightful suggestions improved the final version of this paper. 
Open Access This article is licensed under a Creative Commons Attribution 4.0 International License, which permits use, sharing, adaptation, distribution and reproduction in any medium or format, as long as you give appropriate credit to the original author(s) and the source, provide a link to the Creative Commons licence, and indicate if changes were made. The images or other third party material in this article are included in the article's Creative Commons licence, unless indicated otherwise in a credit line to the material. If material is not included in the article's Creative Commons licence and your intended use is not permitted by statutory regulation or exceeds the permitted use, you will need to obtain permission directly from the copyright holder. To view a copy of this licence, visit http://creativecommons.org/licenses/by/4.0/.

\section{References}

Ansell TJ, Jones PD, Allan RJ et al (2006) Daily mean sea level pressure reconstructions for the European-North Atlantic region for the period 1850-2003. J Clim 19:2717-2742. https://doi.org/10. 1175/JCLI3775.1

Carslaw KS, Lee LA, Reddington CL, Pringle KJ, Rap A, Forster PM, Mann GW, Spracklen DV, Woodhouse MT, Regayre LA, Pierce JR (2013) Large contribution of natural aerosols to uncertainty in indirect forcing. Nature 503:67-71. https://doi.org/10.1038/ nature 12674

Davini P, D'Andrea F (2020) From CMIP3 to CMIP6: Northern Hemisphere atmospheric blocking simulation in present and future climate. J Clim 33(23):10021-10038. https://doi.org/10.1175/ JCLI-D-19-0862.1

Dee DP, Uppala SM, Simmons AJ et al (2011) The ERA-Interim reanalysis: configuration and performance of the data assimilation system. QJR Meteorol Soc 137:553-597. https://doi.org/10.1002/ qj. 828

DEFRA (2016) The costs and impacts of the winter 2013 and 2014 floods: summary. https://www.gov.uk/government/publications/ the-costs-and-impacts-of-the-winter-2013-to-2014-floods

Fabiano F, Christensen H, Strommen K, Athanasiadis P, Baker A, Schiemann R, Corti S (2020) Euro-Atlantic weather Regimes in the PRIMAVERA coupled climate simulations: impact of resolution and mean state biases on model performance. Clim Dyn 54:5031-5048. https://doi.org/10.1007/s00382-020-05271-w

Fabiano F, Meccia VL, Davini P, Ghinassi P, Corti S (2021) A regime view of future atmospheric circulation changes in northern midlatitudes. Weather Clim Dyn 2:163-180. https://doi.org/10.5194/ wcd-2-163-2021

Falkena SK, de Wiljes J, Weisheimer A, Shepherd TG (2020) Revisiting the identification of wintertime atmospheric circulation regimes in the Euro-Atlantic sector. QJR Meteorol Soc 146:28012814. https://doi.org/10.1002/qj.3818

Fenech S, Doherty RM, Heaviside C, Macintyre HL, O'Connor FM, Vardoulakis S, Neal L, Agnew P (2019) Meteorological drivers and mortality associated with $\mathrm{O}_{3}$ and $\mathrm{PM}_{2.5}$ air pollution episodes in the UK in 2006. Atm Environ 213:699-710. https://doi.org/10. 1016/j.atmosenv.2019.06.030

Ferranti L, Corti S (2011) New clustering products. ECMWF Newsl 127:6-11

Ferranti L, Corti S, Janousek M (2015) Flow-dependent verification of the ECMWF ensemble over the Euro-Atlantic sector. QJR Meteorol Soc 141:916-924. https://doi.org/10.1002/qj.2411

Harvey B, Cook P, Shaffrey L, Schiemann R (2020) The response of the northern hemisphere storm tracks and jet streams to climate change in the CMIP3, CMIP5, and CMIP6 climate models. J Geophys Res Atmos. https://doi.org/10.1029/2020JD032701
Hersbach H, Bell B, Berrosfprd P et al (2020) The ERA5 global reanalysis. Q J R Meteorol Soc 146:1999-2049. https://doi.org/10. 1002/qj.3803

Huang WTK, Charlton-Perez A, Lee RW, Neal R, Sarran C, Sun T (2020) Weather regimes and patterns associated with temperaturerelated excess mortality in the UK: a pathway to sub-seasonal risk forecasting. Environ Res Letts 15:124052. https://doi.org/10.1088/ 1748-9326/abcbba

Hurtt GC, Chini LP, Frolking S et al (2011) Harmonization of land-use scenarios for the period 1500-2100: 600 years of global gridded annual land-use transitions, wood harvest, and resulting secondary lands. Clim Change 109:117-161. https://doi.org/10.1007/ s10584-011-0153-2

Jack CD, Jones R, Burgin L, Daron J (2020) Climate risk narratives: an iterative reflective process for coproducing and integrating climate knowledge. Clim Risk Manag 29:100239. https://doi. org/10.1016/j.crm.2020.100239

Kendon M, Sexton D, McCarthy M (2020) A temperature of $20^{\circ} \mathrm{C}$ in the UK winter: a sign of the future? Weather 75(10):318-324. https://doi.org/10.1002/wea.3811

Kendon E, Fosser G, Murphy J et al (2019) UKCP Convection-permitting model projections: science report. https://www.metof fice.gov.uk/pub/data/weather/uk/ukcp18/science-reports/UKCPConvection-permitting-model-projections-report.pdf

Knight JR, Maidens A, Watson PAG, Andrews M, Belcher S, Brunet G, Fereday D, Folland CK, Scaife AA, Slingo J (2017) Global meteorological influences on the record UK rainfall of winter 2013-14. Environ Res Lett. https://doi.org/10.1088/1748-9326/ aa693c

Lamb HH (1972) British Isles weather types and a register of the daily sequence of circulation patterns 1861-1971. Geophys Mem 116:85

Lowe JA, Bernie D, Bett PE et al (2018) UKCP18 Science Overview report. Met Office. https://www.metoffice.gov.uk/pub/data/weath er/uk/ukcp18/science-reports/UKCP18-Overview-report.pdf

Macintyre HL, Heaviside C (2019) Potential benefits of cool roofs in reducing heat-related mortality during heatwaves in a European city. Environ Int 127:430-441. https://doi.org/10.1016/j.envint. 2019.02.065

Macintyre HL, Heaviside C, Taylor J, Picetti R, Symonds P, Cai XM, Vardoulakis S (2018) Assessing urban population vulnerability and environmental risks across an urban area during heatwaves - implications for health protection. Sci Total Environ 610 611:678-690. https://doi.org/10.1016/j.scitotenv.2017.08.062

Marshall GJ (2021) Decadal variability in the impact of atmospheric circulation patterns on the winter climate of Northern Russia. J Clim 34:1005-2021. https://doi.org/10.1175/JCLI-D-20-0566.1

McCarthy M, Armstrong L, Armstrong N (2019) A new heatwave definition for the UK. Weather 74(11):382-387. https://doi.org/ 10.1002/wea.3629

McSweene C, Yamazaki K (2020) UKCP European Circulation Indices: Winter Atlantic Pressure Gradient (North Atlantic Oscillation-NAO). UKCP Factsheet. Met Office. https://www.metoffice. gov.uk/binaries/content/assets/metofficegovuk/pdf/research/ukcp/ ukcp18_factsheet_nao.pdf

McSweeney CF, Jones RG, Lee RW, Rowell DP (2015) Selecting CMIP5 GCMs for downscaling over multiple regions. Clim Dyn 44:3237-3260. https://doi.org/10.1007/s00382-014-2418-8

McSweeneyMcSweeney C, Bett P (2020) UKCP European Circulation Indices: Jet Stream Position and Strength. UKCP Factsheet. Met Office. https://www.metoffice.gov.uk/binaries/content/assets/metof ficegovuk/pdf/research/ukcp/ukcp18_factsheet_jet_stream.pdf

Met Office Hadley Centre (2018) UKCP18 Global Climate Model Projections for the entire globe. Centre for Environmental Data Analysis. http://catalogue.ceda.ac.uk/uuid/f1a2fc3c120f400396a9 2f5de84d596a 
Michelangeli PA, Vautard R, Legras B (1995) Weather regimes: recurrence and quasi stationarity. J Atmos Sci 52:1237-1256. https:// doi.org/10.1175/1520-0469(1995)052\%3c1237:WRRAQS\% 3e2.0.CO;2

Murphy JM, Harris GR, Sexton DMH et al (2018) UKCP18 Land Projections: Science Report. Met Office. https://www.metoffice.gov. uk/pub/data/weather/uk/ukcp18/science-reports/UKCP18-Landreport.pdf

Neal R, Fereday D, Crocket R, Comer R (2016) A flexible approach to defining weather patterns and their application in weather forecasting over Europe. Meteorol Appl 23:389-400. https://doi.org/ 10.1002/met. 1563

Neal R, Dankers R, Saulter A, Lane A, Millard J, Robbins G, Price D (2018) Use of probabilistic medium- to long-range weather-pattern forecasts for identifying periods with an increased likelihood of coastal flooding around the UK. Meteorol Appl 25:534-547. https://doi.org/10.1002/met.1719

Osbourne R, Evans N (2019) Friend or foe? UK farmers' relationships with the weather. J Rural Stud 72:205-215. https://doi.org/10. 1016/j.jrurstud.2019.10.028

Palin EJ, Thornton HE, Mathison CT, McCarthy RE, Clark RT, Dora J (2013) Future projections of temperature-related climate change impacts on the railway network of Great Britain. Clim Change 120:71-93. https://doi.org/10.1007/s10584-013-0810-8

Palin EJ, Scaife AA, Wallace E, Pope ECD, Arribas A, Brookshaw A (2016) Skillful seasonal forecasts of winter disruption to the UK transport system. J Appl Meteorol Climatol 55:325-344. https:// doi.org/10.1175/JAMC-D-15-0102.1

Panteli M, Mancarella P (2015) Influence of extreme weather and climate change on the resilience of power systems: impacts and possible mitigation strategies. Electr Power Syst Res 127:259-270. https://doi.org/10.1016/j.epsr.2015.06.012

Papritz L, Grams CM (2018) Linking low-frequency large-scale circulation patterns to cold air outbreak formation in the northeastern North Atlantic. Geophys Res Letts 45:2542-2553. https://doi.org/ 10.1002/2017GL076921

Pearson K (1900) On the criterion that a given system of deviations from the probable in the case of a correlated system of variables is such that it can be reasonably supposed to have arisen from random sampling. Lond Edinb Dublin Philos Mag J Sci 50(302):157175. https://doi.org/10.1080/14786440009463897

Peings Y, Simpkins G, Magnusdottir G (2016) Multidecadal fluctuations of the North Atlantic Ocean and feedback on the winter climate in CMIP5 control simulations. J Geophys Res Atmos 121:2571-2592. https://doi.org/10.1002/2015JD024107

Peings Y, Cattiaux J, Vavrus SJ, Magnusdottir G (2018) Projected squeezing of the wintertime North-Atlantic jet. Environ Res Lett 13:074016. https://doi.org/10.1088/1748-9326/aacc79

Philipp A, Jacobeit J, Della-Marta PM et al (2007) Long-term variability of daily North Atlantic-European pressure patterns since 1850 classified by simulated annealing clustering. J Clim 20:40654095. https://doi.org/10.1175/JCLI4175.1
Pregnolato M, Ford A, Robson C, Glenis V, Barr S, Dawson RJ (2016) Assessing urban strategies for reducing the impacts of extreme weather on infrastructure networks. R Soc Open Sci 3(5):1-15. https://doi.org/10.1098/rsos.160023

Richardson D, Fowler HJ, Kilsby CG, Neal R (2017) A new precipitation and drought climatology based on weather patterns. Int $\mathrm{J}$ Clim 38(2):630-648. https://doi.org/10.1002/joc.5199

Ridley JK, Blockley EW, Keen AB, Rae JGL, West AE, Schroeder D (2017) The sea ice model component of HadGEM3-GC3.1. Geosci Model Dev 11:713-723. https://doi.org/10.5194/ gmd-11-713-2018

Sexton DMH, McSweeney CF, Rostron JW et al (2021) A perturbed parameter ensemble of HadGEM3-GC3.05 coupled model projections: part 1: selecting the parameter combinations. Clim Dyn. https://doi.org/10.1007/s00382-021-05709-9

Shepherd TG, Boyd E, Calel RA et al (2018) Storylines: an alternative approach to presenting uncertain in physical aspects of climate change. Clim Change 151:555-571. https://doi.org/10.1007/ s10584-018-2317-9

Storkey D, Megann A, Mathiot P, Sinha B, Calvert D, Hewitt H, Blaker A, Kuhlbrodt T, Graham T, Hyder P (2017) UK Global Ocean GO6 and GO7: a traceable hierarchy of model resolutions. Geosci Model Dev 11:3187-3213. https://doi.org/10.5194/gmd-2017-263

Taylor KE, Stouffer RJ, Meehl GA (2012) An overview of CMIP5 and the experiment design. Bull Am Meteorol Soc 93:485-498. https://doi.org/10.1175/BAMS-D-11-00094.1

van Vuuren DP, Edmonds J, Kainuma M et al (2011) The representative concentration pathways: an overview. Clim Change 109:5-31. https://doi.org/10.1007/s10584-011-0148-z

Walters DN, Baran AJ, Boutle I et al (2017) The Met Office Unified Model Global Atmosphere 7.0/7.1 and JULES Global Land 7.0 configurations. Geosci Model Dev 12:1909-1963. https://doi.org/ 10.5194/gmd-12-1909-2019

Williams KD, Copsey D, Blockley EW et al (2017) The Met Office Global Coupled model 3.0 and 3.1 (GC3 and GC3.1) configurations. J Adv Model Earth Syst 10:357-380. https://doi.org/10. 1002/2017MS001115

Yamazaki K, Sexton DMH, Rostron JW et al (2021) A perturbed parameter ensemble of HadGEM3-GC3.05 coupled model projections: part 2: global performance and future changes. Clim Dyn. https://doi.org/10.1007/s00382-020-05608-5

Zappa G, Shepherd TG (2017) Storylines of atmospheric circulation change for European regional climate impact assessment. J Clim 30:6561-6577. https://doi.org/10.1175/JCLI-D-16-0807.1

Publisher's Note Springer Nature remains neutral with regard to jurisdictional claims in published maps and institutional affiliations. 